\title{
Study the Efficacy of Sorafenib, Vemurafenib and Dabrafenib on BRAF Wild and Mutated Genes Using Computational
}

\section{Approach}

\author{
Alla $\mathrm{MH}^{1}$, Enas $\mathrm{AS}^{1}$, Asia $\mathrm{M}^{1}$, Salah $\mathrm{MB}^{1}$, Anton $\mathrm{H}^{2}$ and Abdul MG ${ }^{1 *}$ \\ ${ }^{1}$ Department of Medicinal Chemistry, Faculty of Pharmacy, University of Tripoli, \\ Austria \\ ${ }^{2}$ Department of Biosciences, University of Salzburg, Austria
}

\section{Short Communication}

Volume 3 Issue 2

Received Date: June 22, 2018

Published Date: June 26, 2018

*Corresponding author: Abdul M Gbaj, Department of Medicinal Chemistry, Faculty of Pharmacy, University of Tripoli, Austria, Tel: +218913556785; Email: abdulgbaj1@hotmail.com

\section{Abstract}

The BRAF gene provides instructions for making a protein that helps transmit chemical signals from outside the cell to the cell's nucleus. Activation of the BRAF/MAPK kinase (MEK)/extracellular signal-regulated kinase (ERK) pathway has been shown to increase tumor cell proliferation, survival, invasion, and tumor angiogenesis. The BRAF gene is an oncogene and when it is mutated it turns the normal cells to become cancerous. Molecular docking showed that sorafenib, vemurafenib and dabrafenib are inhibitors that have the potential to inhibit the activity of many tumours related to BRAF mutations. In conclusion, BRAF inhibitors, such as Sorafenib, vemurafenib and dabrafenib, are specifically active against BRAF-mutant carcinoma.

Keywords: BRAF; Tumour; Sorafenib; Vemurafenib; Dabrafenib

\section{Introduction}

B-Raf gene is a member of the Raf kinase family and is a downstream target of RAS, and it has a crucial function in the MAPK/ERK signalling pathway. The MAPK/ERK pathway (also recognized as the Ras-Raf-MEK-ERK pathway) is a chain of proteins in the cell that converses a signal from a receptor on the exterior of the cell to the DNA in the nucleus of the cell [1]. The BRAF gene is positioned on the long arm of chromosome 7 (7q34) and codes for the serine/threonine protein kinase, B-Raf [2,3]. There are around 30 mutations of the BRAF gene related to the human and these mutations are mostly located within the part of the gene encoding the kinase domain, clustered to two regions containing the glycine-rich $\mathrm{P}$ loop of the $\mathrm{N}$-lobe and the activation segment [4,5]. One of these mutations, the V600E (T1799A), resulting in a T-toA transversion at position 1799 with a valine-to- 


\section{Advances in Pharmacology and Clinical Trials}

glutamate substitution, and it is the most widespread BRAF mutation in papillary thyroid cancers, renal cell carcinoma and hepatocellular carcinoma, anaplastic thyroid cancers and other types of tumours [6,7]. Sorafenib, vemurafenib and dabrafenib are multi-targeted kinase inhibitors with established activity on many types of tumours. They have shown an activity as anti-BRAF agents mainly on mutated BRAF V600E than wild-type. The aim of this study was to identify the strength of the binding energy of sorafenib, vemurafenib and dabrafenib as BRAF inhibitors with mutated and wild-type BRAF gene using molecular docking.

\section{Materials and Methods}

\section{Molecular Docking}

The computational studies were performed in order to elucidate the binding energies of sorafenib, vemurafenib and dabrafenib to the BRAF gene. The 2D structures of sorafenib, vemurafenib and dabrafenib were drawn using ChemDraw Ultra (version 8.0, Cambridgesoft Com., USA). Chem3D Ultra was used to convert 2D structure into 3D and the energy was minimized using the semi-empirical AM1 method. Minimized energy to minimum RMS gradient of 0.100 was set in each iteration. All structures were saved as PDB file format for input to ADT. All AOLE constituents chemical structures were then saved in PDBQT file format to carry out docking in ADT. The crystal structures of BRAF gene in a complex with a transition-state analogue were downloaded from the Protein Data Bank https://www.rcsb.org/structure/3q4c. The molecular docking of sorafenib, vemurafenib and dabrafenib were accomplished by AutoDock 4.2 software from the Scripps Research Institute (TSRI) (http://autodock.scripps.edu/). Firstly, the polar hydrogen atoms were added into BRAF gene molecules. Then, the partial atomic charges of BRAF gene and sorafenib, vemurafenib and dabrafenib molecules were calculated using Kollman methods [8]. In the process of molecular docking, the grid maps of dimensions ( $62 \AA \mathrm{X}$ $62 \AA$ X $62 \AA$ ) with a grid-point spacing of $0.376 \AA$ and the grid box centered. The number of genetic algorithm runs and the number of evaluations was set to 100 . All other parameters were default settings. Cluster analysis was performed on the results of docking by using a root mean square (RMS) tolerance of $2.0 \AA$, dependent on the binding free energy. Lastly, the dominating configuration of the binding complex of sorafenib, vemurafenib and dabrafenib and BRAF gene proteins with minimum energy of binding determined.

\section{Results and Discussion}

Table 1 shows the energies of binding of the sorafenib, vemurafenib and dabrafenib with BRAF gene obtained by using molecular docking strategy.

\begin{tabular}{|c|c|c|}
\hline Inhibitor name & $\begin{array}{c}\text { Binding energy with wild type BRAF (Protein Data Bank } \\
\text { under accession number 30G7) }\end{array}$ & $\begin{array}{c}\text { Binding energy with mutated BRAF } \\
\text { v600E (Protein Data Bank under } \\
\text { accession number 3q4c) }\end{array}$ \\
\hline Sorafenib & -6.48 & -8.51 \\
\hline Dabrafenib & -7.33 & -8.28 \\
\hline Vemurafenib & -8.41 & -10.2 \\
\hline
\end{tabular}

Table 1: Various energies in the binding process of-sorafenib, vemurafenib and dabrafenib with BRAF gene obtained from molecular docking. The unit of all energies was $\mathrm{kJ} / \mathrm{mol}$.

a $\Delta \mathrm{G}$ is the free binding energy change in the binding process ( $\mathrm{Kcal} / \mathrm{mole})$.

Our modeling study indicates substantial interactions between sorafenib, vemurafenib and dabrafenib with BRAF gene. The docking binding energies are shown in Table 1. The sorafenib, vemurafenib and dabrafenib were able to form hydrogen bonds, pi-pi stacking and Pi-alkyl interaction with many amino acids residues. Table 1 suggests that sorafenib, vemurafenib and dabrafenib could be more effective in BRAF-mutated tumors than that of BRAF-wild tumors. It has been reported that sorafenib is active in gastrointestinal stromal tumors (GISTs) which are resistant to imatinib, sunitinib and nilotinib due to the BRAF mutations [9]. It has been also reported that vemurafenib is an oral BRAF inhibitor and it is selective for the V600E mutation, and has been proven to be effective in treating advanced melanoma patients with the same mutation[10]. The obtained molecular modeling results are consistent with the literature in which Gardini et al. found that sorafenib has a good response in lung lesions, carrying the BRAF mutation, whereas no response was observed in the hepatic lesion, which was BRAF wild type [11]. It has been also reported that vemurafenib is an oral BRAF inhibitor and it is selective for the V600E mutation, and has been proven to be effective in treating advanced melanoma patients with 


\section{Advances in Pharmacology and Clinical Trials}

the same mutation. In conclusion, all these literature evidnaces indicate that sorafenib, vemurafenib and dabrafenib are more effective on BRAF-mutated tumors than that of BRAF-wild tumors and were consistent with our modeling results.

\section{References}

1. Fang G, Chen S, Huang Q, Chen L, Liao D (2018) Curcumin suppresses cardiac fibroblasts activities by regulating the proliferation and cell cycle via the inhibition of the p38 MAPK/ERK signaling pathway. Mol Med Rep.

2. Hagemann C, Rapp UR (1999) Isotype-specific functions of Raf kinases. Exp Cell Res 253(1): 34-46.

3. Feller SM, Posern G, Voss J, Kardinal C, Sakkab D, et al. (1998) Physiological signals and oncogenesis mediated through Crk family adapter proteins. J Cell Physiol 177(4): 535-552.

4. Honecker F, Wermann H, Mayer F, Gillis AJ, Stoop H, et al. (2009) Microsatellite instability, mismatch repair deficiency, and BRAF mutation in treatmentresistant germ cell tumors. J Clin Oncol 27(13): 21292136.

5. Cameselle TJ, Abdulkader I, Perez BR, Vazquez BA, Alberte LL, et al. (2009) BRAF mutation in solid cell nest hyperplasia associated with papillary thyroid carcinoma. A precursor lesion? Hum Pathol 40(7): 1029-1035.
6. Li Q, Jin WX, Jin YX, Zheng ZC, Zhou XF, et al. (2018) Clinical effect of MUC1 and its relevance to BRAF V600E mutation in papillary thyroid carcinoma: a case-control study. Cancer Manag Res 10: 1351-1358.

7. Asl JM, Almasi S, Tabatabaiefar MA (2014) High frequency of BRAF proto-oncogene hot spot mutation V600E in cohort of colorectal cancer patients from Ahvaz City, southwest Iran. Pak J Biol Sci 17(4): 565569.

8. Tiwari R, Mahasenan K, Pavlovicz R, Li C, Tjarks W (2009) Carborane clusters in computational drug design: a comparative docking evaluation using AutoDock, FlexX, Glide, and Surflex. J Chem Inf Model 49(6): 1581-1589.

9. Agaram NP, Wong GC, Guo T, Maki RG, Singer S, et al. (2008) Novel V600E BRAF Mutations in ImatinibNaive and Imatinib-Resistant Gastrointestinal Stromal Tumors. Genes Chromosomes Cancer 47(10): 853-859.

10. Villaruz LC, Burns TF, Ramfidis VS, Socinski MA (2013) Personalizing Therapy in Advanced Nonâ€"Small Cell Lung Cancer. Semin Respir Crit Care Med 34(6): 822-836.

11. Casadei GA, Chiadini E, Faloppi L, Marisi G, Delmonte A, et al. (2016) Efficacy of sorafenib in BRAF-mutated non-small-cell lung cancer (NSCLC) and no response in synchronous BRAF wild type-hepatocellular carcinoma: a case report. BMC Cancer 16.

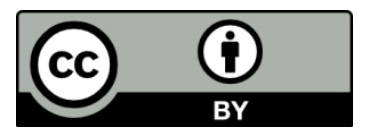

